\title{
Modelo de decisión multicriterio difuso para la selección de contratistas en proyectos de infraestructura: caso Colombia
}

\section{A fuzzy multi-criteria decision-making model for infrastructure projects contractor selection: the Colombian case}

Fecha de entrega: 2 de junio 2016 Fecha de aceptación: 12 de septiembre 2016

\section{Julián Mayor ${ }^{1}$, Sergio Botero² y Juan David González-Ruiz ${ }^{3}$}

${ }^{1}$ Constructora Conconcreto S.A. Procesos Contables, Medellín, Colombia, jamayorr@unal.edu.co

${ }^{2}$ Modelamiento y Análisis, Energía, Ambiente y Economía, Facultad de Minas, Bloque M8B, oficina 106, Universidad Nacional de Colombia, Carrera 80 No.65-223, Medellín, Colombia, sbotero@unal.edu.co

${ }^{3}$ Dirección de Empresas, Institución Universitaria Esumer, Universidad Nacional de Colombia, Calle 76 No. 80-126, Bloque 1 Piso 2, Medellín, Colombia,jdgonzalez@esumer.edu.co,jdgonza3@unal.edu.co

Los métodos de decisión multicriterio son cada vez más útiles para solucionar problemas de selección de contratistas de construcción e infraestructura debido al aumento de la comprensión de su utilidad. La investigación propone un modelo multicriterio de selección de contratistas para proyectos de infraestructura de iniciativa pública en Colombia. Para ello se revisó los métodos correspondientes en las diferentes etapas; es decir, la selección de criterios, la ponderación de criterios, la precalificación y selección final. El modelo combina la precalificación con la selección, integración que es más eficaz en la búsqueda del contratista más competente. Los criterios de precalificación se clasifican en 4 categorías: atributos de experiencia, técnicos, organizacionales y financieros y/o económicos, incorporando subcriterios de dimensión cualitativa y cuantitativa. Los criterios de selección son 4: precalificación, oferta técnica, oferta de calidad y oferta económica. Para determinar los criterios y sus respectivos pesos se entrevistaron expertos en proyectos de infraestructura, quienes observan el proceso desde diferentes puntos de vista: de la Agencia Nacional de Infraestructura, de una banca de inversión, un académico y de una constructora que desarrolla proyectos de infraestructura.

Palabras clave: método TOPSIS, lógica difusa, proyectos de infraestructura, selección de contratistas
Multicriteria decision methods have become increasingly useful for troubleshooting the selection of construction contractors and infrastructure due to the increased understanding of its usefulness. This research proposes a model of multi-criterial of contractors for infrastructure projects of public initiative in Colombia. To do this the corresponding methods at different stages were revised; that is, the selection criteria, weighting criteria, prequalification and final selection. The model combines the prequalification selection; integration is more effective in finding the most competent contractor. The prequalification criteria have been classified into four attribute categories: experience, technical, organizational and financial and/or economic, sub incorporating qualitative and quantitative dimension. The selection criteria are 4: pre-qualification, technical offer, quality offer and financial offer. To determine the criteria and their respective weights experts were interviewed in infrastructure projects, who observe the process from different viewpoints, that is, from the National Infrastructure Agency, from an investment bank, an academic and from a construction company that develops infrastructure projects.

Keywords: TOPSIS method, fuzzy logic, infrastructure project, contractor selection

\section{Introducción}

Los proyectos de infraestructura, en especial de transporte, son de vital importancia para el crecimiento económico de un país, dado que son los medios que soportan el intercambio de productos y servicios. Muchos proyectos son complejos, requieren grandes inversiones y en muchos casos un alto nivel de experticia en las diferentes disciplinas de la ingeniería. Además de la complejidad, en los procesos de adjudicación pueden ocurrir situaciones no deseadas como favoritismo, corrupción o elementos 
subjetivos que conlleven a una elección errónea del contratista. Por lo tanto, la adjudicación de contratos en proyectos de infraestructura, requiere especial atención porque implica un alto nivel de riesgo que compromete no solo el éxito de los proyectos, sino también recursos públicos, desarrollo social y crecimiento de un país. Los procesos de adjudicación de proyectos de infraestructura normalmente se establecen mediante licitación pública donde se exponen los criterios de preselección y selección. Generalmente en la primera etapa, se exponen los criterios mínimos de cumplimiento y, por lo tanto, son descartados aquellos contratistas que no los cumplan. En la segunda etapa, se adjudica el contrato de acuerdo al principio de aceptación de la oferta de precio más bajo (Jato-Espino et at., 2014a; Palaneeswaran y Kumaraswamy, 2001; Topcu, 2004). Sin embargo, la evaluación con base en el precio más bajo es una de las principales causas de problemas de entrega de proyectos (Hatush y Skitmore, 1998; NietoMorote y Ruz-Vila, 2012; Singh y Tiong, 2005).

Algunos países, entre ellos Colombia, han corregido el problema de otorgar el contrato por la oferta más baja, implementando mecanismos de adjudicación como la media aritmética o la media geométrica ajustada (ANI, 2013). Sin embargo, en Colombia, muchos proyectos de infraestructura están retrasados en la entrega de las obras, algunas con dos o tres años, otros, aunque no se han vencido tienen porcentajes de avance inferiores al 50\%; en estos proyectos, el principal factor del retraso es el incumplimiento por parte del contratista (líos de ejecución, mal que aqueja varias obras, 2014, http://www.eltiempo. $\mathrm{com} /$ ). Probablemente este problema se genera porque en estos procesos de selección se evalúa la experiencia en inversión del contratista y no necesariamente la competencia y experticia en el campo específico de la ingeniería. Esto sucede porque se desarticula el proceso de preselección con la adjudicación; por lo tanto, los resultados del proceso solo sirven para descartar alternativas y no como un insumo para el proceso de adjudicación, dado que todos los candidatos preseleccionados empiezan en igualdad de condiciones en la etapa de selección desconociendo el desempeño individual en esta primera etapa. De esta manera, la complejidad en los procesos de adjudicación se debe fundamentar en evaluar las capacidades de los contratistas para cumplir el contrato y ante situaciones adversas tales como factores económicos, tecnológicos, ambientales y otros que pueden afectar el entorno interno y externo de los proyectos y contratistas. Al mismo tiempo, desde la óptica del contratante, el proceso de adjudicación debe tener como objetivo la obtención de bienes y servicios al precio más bajo posible al estimular la competencia y evitar el favoritismo (Ballesteros-Perez et al., 2013). Así, el proceso de Toma de Decisiones de Adjudicación de Contratos de Infraestructura TDACI debe ser un instrumento que promueva la transparencia y no permita la corrupción.

En consecuencia, las complejidades mencionadas con anterioridad pueden ser reducidas por los Métodos de Decisión Multicriterio MCDM. En esta línea, la Toma de Decisiones Multicriterio TDM, se define como el proceso que se encarga de diseñar la mejor alternativa de selección o de seleccionar la mejor entre un conjunto de alternativas, la cual utiliza los atributos generales más atractivos e involucra la selección de la alternativa óptima por medio de modelos de preferencia (Turskis, 2008). Así mismo, los MCDM son un enfoque de apoyo en la evaluación y decisión operativa para hacer frente a problemas complejos que ofrecen una alta incertidumbre, objetivos contradictorios, diferentes formas de datos e información, intereses múltiples y perspectivas, evolución de sistemas complejos. En comparación con el enfoque único criterio, la ventaja distintiva de métodos MCDM es emplear múltiples criterios o atributos para obtener un resultado de toma de decisiones integrado (Wang et at., 2009). Por lo tanto, seleccionar el contratista más competente debe ser el objetivo principal en un proceso de adjudicación dado que esto puede tener como consecuencia el mejoramiento del desempeño general de los demás participantes del proyecto. Así, elegir el contratista adecuado para un trabajo influye en la calidad del trabajo, así como en el progreso en los niveles y calidad de la construcción, por lo tanto, en el proceso de licitación la selección óptima del contratista es vital para una oferta precisa y realista (Plebankiewicz, 2012).

Con el objetivo de proponer una metodología para seleccionar el contratista más competente, el artículo plantea un modelo multicriterio de selección de contratistas para proyectos de infraestructura con aplicación para el contexto colombiano, el cual integra los procesos de precalificación y selección. Para el desarrollo de la propuesta se revisó la literatura publicada en el periodo 
2000-2014. La metodología propuesta incluye entrevistas con expertos que proporcionaron información fundamental para la selección de criterios y su respectiva asignación de pesos. El modelo es validado con una simulación de un proceso de contratación pública donde se podrán contrastar los resultados y establecer conclusiones y recomendaciones que sirvan de mejoramiento al proceso de selección de contratistas para proyectos de infraestructura en Colombia.

El artículo está estructurado de la siguiente manera. Después de la introducción, se presentan las principales modalidades de contratación. Luego se introduce los métodos multicriterio con su respectivo enfoque y su aplicación en la selección de contratistas de infraestructura, sirviendo como base para proponer la metodología, la cual es presentada en la siguiente sección y validada con un caso de estudio. Además, se presenta el proceso de selección de contratistas en Colombia, como eje fundamental de la metodología propuesta. Finalmente, se presentan las conclusiones y futuros trabajos.

\section{Contratación pública para proyectos de infraestructura}

Alrededor del mundo se utilizan diferentes modalidades para la selección de contratistas para desarrollar proyectos de infraestructura, entre ellos se destacan la licitación abierta, licitación selectiva o restringida, precalificación o negociación (Palaneeswaran y Kumaraswamy, 2001; Topcu, 2004). De acuerdo a las particularidades del proyecto, el contratante convoca y utiliza la modalidad más pertinente. Una de las modalidades de selección de contratista más utilizadas es licitación pública. En este método de contratación, los oferentes a través de mecanismos de publicación de los pliegos conocen el alcance, especificaciones y términos, así como los criterios mediante los cuales se evaluarán las ofertas. La Figura 1 muestra las principales etapas en los procesos de contratación pública.

Por otra parte, existe una amplia variación en los procedimientos de selección, por ejemplo; algunas subastas invitan solo a compañías nacionales, mientras que otras aceptan competidores internacionales. La diferencia más importante en el diseño de la contratación pública es la importancia de la relación calidad-precio. Algunas subastas siguen estrictamente los criterios de menor precio, pero otros consideran la variable calidad (Estache e Iimi, 2011). En la Tabla 1 se puede observar que algunos países han modificado sus regulaciones en cuanto a seleccionar la oferta con menor precio, utilizando otras modalidades de selección o exclusión, métodos que combinan precio y calidad en una puntuación total o por ejemplo excluyendo las ofertas que consideran anormalmente bajas, o las ofertas más altas y más bajas; entre tanto otros seleccionan la oferta más cercana a la media.

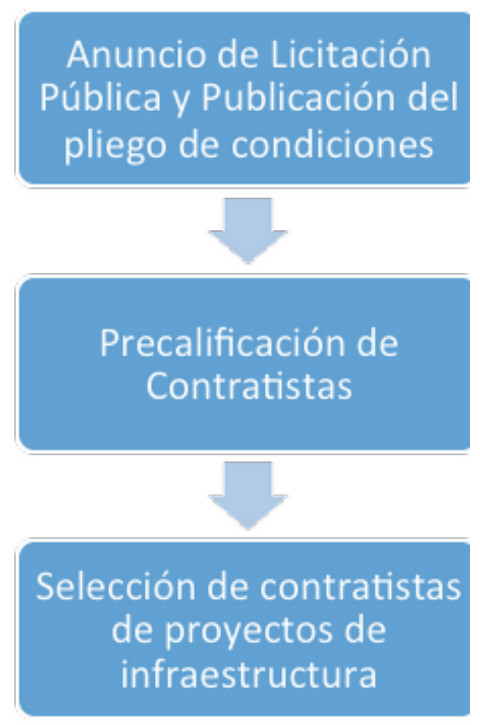

Figura 1: Principales etapas del proceso de contratación pública.

\section{Métodos de Decisión Multicriterio MCDM}

Para desarrollar la propuesta metodológica para seleccionar el mejor contratista, la TDM juega un rol importante en diseño, se define como el proceso que se encarga de diseñar la mejor alternativa de selección. La metodología de los métodos multicriterio consiste en determinar criterios y subcriterios de evaluación, asignar los pesos respectivos a cada criterio y subcriterio, los cuales se analizan a través de comparaciones pareadas para medir la relación entre criterios y su importancia. Posteriormente expertos evalúan las alternativas por cada criterio, asignando una calificación y así obtener la alternativa óptima para el problema de acuerdo al criterio profesional de los expertos. La Tabla 2 muestra una descripción general de los métodos multicriterio existentes de acuerdo a su enfoque. 
Tabla 1: Modelos de selección y de exclusión de contratistas en procesos de licitación

\begin{tabular}{|c|c|c|c|}
\hline País & $\begin{array}{c}\text { Modalidades de } \\
\text { selección }\end{array}$ & $\begin{array}{c}\text { Modalidad de } \\
\text { descarte }\end{array}$ & Referencias \\
\hline $\begin{array}{l}\text { U n i ó n } \\
\text { Europea }\end{array}$ & $\begin{array}{l}\text { Precio más bajo- } \\
\text { EMAT-oferta } \\
\text { más cercana al } \\
\text { promedio }\end{array}$ & $\begin{array}{l}\text { Rechaza } \\
\text { ofertas más } \\
\text { bajas y más } \\
\text { altas }\end{array}$ & $\begin{array}{c}\text { Bergman y } \\
\text { Lundberg (2013) } \\
\text { Estache e Iimi } \\
(2011) \\
\text { Lambropoulos } \\
(2007)\end{array}$ \\
\hline Perú & $\begin{array}{l}\text { Oferta más } \\
\text { cercana al } \\
\text { promedio }\end{array}$ & $\begin{array}{c}\text { Rechaza } \\
\text { ofertas más } \\
\text { bajas y más } \\
\text { altas }\end{array}$ & $\begin{array}{l}\text { Lambropoulos } \\
\text { (2007) }\end{array}$ \\
\hline Corea & $\begin{array}{l}\text { Oferta más } \\
\text { cercana al } \\
\text { promedio }\end{array}$ & $\begin{array}{c}\text { Rechaza } \\
\text { ofertas más } \\
\text { bajas y más } \\
\text { altas }\end{array}$ & $\begin{array}{l}\text { Lambropoulos } \\
\text { (2007) }\end{array}$ \\
\hline Taiwán & $\begin{array}{l}\text { EMAT-precio } \\
\text { calidad }\end{array}$ & $\begin{array}{c}\text { Rechaza } \\
\text { ofertas más } \\
\text { bajas y más } \\
\text { altas }\end{array}$ & $\begin{array}{l}\text { Wang et al. } \\
\text { (2013) }\end{array}$ \\
\hline China & $\begin{array}{c}\text { Precio más } \\
\text { bajo- oferta más } \\
\text { favorable precio } \\
\text { calidad }\end{array}$ & $\mathrm{N} / \mathrm{A}$ & $\begin{array}{c}\text { Padhi y } \\
\text { Mohapatra (2010) } \\
\text { Wang et al. }(2013) \\
\text { Ye et al. }(2013)\end{array}$ \\
\hline EEUU & $\begin{array}{l}\text { Precio más bajo- } \\
\text { tiempo }\end{array}$ & N/A & $\begin{array}{c}\text { Bastias y } \\
\text { Molenaar (2010) } \\
\text { Lambropoulos } \\
(2007)\end{array}$ \\
\hline India & Precio más bajo & N/A & $\begin{array}{c}\text { Padhi y } \\
\text { Mohapatra (2010) }\end{array}$ \\
\hline Sudáfrica & $\begin{array}{l}\text { EMAT-precio } \\
\text { calidad }\end{array}$ & N/A & Bolton (2009) \\
\hline Turquía & Precio más bajo & N/A & $\begin{array}{c}\text { Palaneeswaran y } \\
\text { Kumaraswamy } \\
(2001), \text { Topcu } \\
(2004)\end{array}$ \\
\hline Chile & $\begin{array}{c}\text { Oferta más } \\
\text { favorable - } \\
\text { precio calidad }\end{array}$ & N/A & MOP (2009) \\
\hline Colombia & $\begin{array}{c}\text { Oferta más } \\
\text { favorable } \\
\text {-precio calidad }\end{array}$ & $\begin{array}{c}\text { Establece un } \\
\text { límite inferior } \\
\text { y descarta las } \\
\text { ofertas que se } \\
\text { encuentren por } \\
\text { debajo a dicho } \\
\text { límite }\end{array}$ & DPN (2013) \\
\hline
\end{tabular}

\section{Métodos de selección de criterios y asignación de pesos}

El primer paso para realizar la selección de la mejor alternativa es la selección de los criterios y luego asignarle los pesos que determinan la importancia relativa de cada uno respecto al total y, de esta manera, realizar una ponderación de acuerdo al método empleado. Si bien algunas de las metodologías podrían no requerir ponderación de criterios (de hecho, algunas no requieren selección de criterios), en la
Tabla 2: Métodos multicriterio y enfoques (Roy y Słowiński, 2013)

\begin{tabular}{|c|c|c|}
\hline Clasificación & Abreviatura & Método \\
\hline \multirow{12}{*}{$\begin{array}{l}\text { Métodos } \\
\text { basados en } \\
\text { la teoría del } \\
\text { valor }\end{array}$} & AHP & Proceso analítico jerárquico \\
\hline & ANP & Proceso de red analítica \\
\hline & COPRAS & $\begin{array}{l}\text { Evaluación compleja } \\
\text { proporcional }\end{array}$ \\
\hline & FSs & Conjuntos difusos \\
\hline & TOPSIS & $\begin{array}{l}\text { Técnica para el orden de } \\
\text { preferencia por similitud a } \\
\text { solución real }\end{array}$ \\
\hline & VIKOR & $\begin{array}{l}\text { Optimización multidisciplinar y } \\
\text { solución de compromiso }\end{array}$ \\
\hline & UTA & Utilidades aditivas \\
\hline & MCS & Simulación Monte Carlo \\
\hline & UT & Teoría de la utilidad \\
\hline & GST & Teoría del sistema Gris \\
\hline & MAUT & $\begin{array}{l}\text { Teoría de la utilidad } \\
\text { multiatributo }\end{array}$ \\
\hline & MAVT & Teoría del valor multiatributo \\
\hline \multirow{6}{*}{$\begin{array}{l}\text { Métodos de } \\
\text { clasificación }\end{array}$} & PROMETHEE & $\begin{array}{l}\text { Clasificación de preferencia, } \\
\text { método de enriquecimiento de } \\
\text { evaluaciones }\end{array}$ \\
\hline & ELECTRE & $\begin{array}{l}\text { Eliminación y opciones que } \\
\text { reflejan la realidad }\end{array}$ \\
\hline & & $\begin{array}{l}\text { Métodos de regresión ordinal } \\
\text { robusta }\end{array}$ \\
\hline & UTA GMS & $\begin{array}{l}\text { La regresión ordinal robusta para } \\
\text { problemas de clasificación y } \\
\text { elección }\end{array}$ \\
\hline & GRIP & $\begin{array}{lll}\text { Regresión } & \text { generalizada } \\
\text { intensidades de preferencia }\end{array}$ \\
\hline & RUTA & $\begin{array}{l}\text { Método de desagregación de } \\
\text { preferencia }\end{array}$ \\
\hline
\end{tabular}

mayoría de los casos es necesario hacerlo y se convierte en un proceso de gran importancia, pues independientemente del método de selección de contratistas, la ponderación de los criterios indiscutiblemente puede llegar a cambiar la escogencia, por su influencia directa sobre el resultado final. En últimas, lo que se busca al emplear un método para definir la importancia relativa de los criterios es disminuir la subjetividad que implícitamente está en las apreciaciones humanas, para construir una distribución objetiva y consistente. La Tabla 3 presenta algunos de los métodos empleados para asignación de pesos en selección de contratistas en proyectos de construcción o infraestructura.

Los métodos de selección y ponderación de criterios se clasificaron en dos categorías: los métodos subjetivos y los objetivos. Se puede observar que los métodos más populares 
en proyectos de construcción son los AHP y asignación por expertos que hacen parte de la categoría subjetiva. Los pesos de los criterios determinados por los métodos de ponderación subjetivas dependen sólo de la preferencia de quienes toman las decisiones y no de los datos cuantitativos medidos en los proyectos de construcción. Por el contrario, los pesos objetivos se obtienen por métodos matemáticos basados en el análisis de los datos iniciales. Los juicios de quienes toman las decisiones a veces dependen de su conocimiento o información. Por lo tanto, los errores de los pesos de los criterios en cierta medida son inevitables. Como se puede observar, ninguno de los dos enfoques es perfecto. Se puede sugerir que un método integrado podría ser más apropiado para determinar los pesos de criterios (Wang et al., 2009).

Tabla 3: Métodos de selección de criterios y asignación de pesos

\begin{tabular}{|c|c|c|c|}
\hline Categoría & Método & Referencias & $\begin{array}{l}\text { Número } \\
\text { de refer- } \\
\text { encias }\end{array}$ \\
\hline \multirow{3}{*}{ Subjetivo } & $\begin{array}{c}\mathrm{AHP} / \mathrm{AHP} \\
\text { difuso }\end{array}$ & $\begin{array}{l}\text { Anagnostopoulos y Vavatsikos } \\
\text { (2006), Chou et al. (2013), } \\
\text { Jaskowski et al. (2010), Liu y } \\
\text { Yan (2007), Nassar y Hosny } \\
\text { (2013), Park y Chul (2012), San } \\
\text { Cristobal (2012), Topcu (2004), } \\
\text { Trivedi (2011), Wang et al. } \\
(2013,2013 b)\end{array}$ & 11 \\
\hline & ANP & El-Abbasy et al. (2013) & 1 \\
\hline & $\begin{array}{l}\text { Asignación } \\
\text { por } \\
\text { expertos / } \\
\text { tomadores } \\
\text { de decisión }\end{array}$ & $\begin{array}{l}\text { Alhumaidi (2015), Awad y } \\
\text { Fayek (2012), Bendana et al. } \\
\text { (2008), Darvish et al. (2009), } \\
\text { Horta et al. (2013), Li et al. } \\
\text { (2007), Nieto-Morote y Ruz- } \\
\text { Vila (2012), Plebankiewicz } \\
\text { (2009, 2012, 2014), Wei et al. } \\
\text { (2011), Singh y Tiong (2015), } \\
\text { Vahdani et al. (2009) }\end{array}$ & 13 \\
\hline \multirow{2}{*}{ Objetiva } & $\begin{array}{l}\text { Pesos } \\
\text { iguales }\end{array}$ & Arslan et al. (2008) & 1 \\
\hline & Entropía & Wang et al. (2009b) & 1 \\
\hline
\end{tabular}

\section{Criterios de precalificación y selección}

Las literaturas revisadas son tanto teórica, conceptual y empírica. Así, esta investigación se enfoca principalmente en los Métodos Multicriterios MCDM aplicados a los procesos de adjudicación de contratos de infraestructura donde el contratante del proyecto es un organismo público. La multiplicidad de criterios y herramientas de medición que se está desarrollando en este campo de rápido crecimiento demuestra la importancia de la labor conceptual y metodológica en esta área. El desarrollo y selección de criterios requieren parámetros relacionados con la confiabilidad, conveniencia, practicidad y limitaciones de la medición. Los criterios utilizados para seleccionar contratistas de infraestructura y construcción en la literatura se dividen principalmente en cuatro atributos: experiencia, técnicos, organizacionales y financieros y económicos, que se resumen en la Tabla 4.

Tabla 4: Principales categorías de evaluación de criterios (Watt et al., 2009)

\begin{tabular}{|c|c|}
\hline Atributos & Criterio \\
\hline \multirow{4}{*}{ Experiencia } & Experiencia \\
\hline & $\begin{array}{l}\text { Desempeño en el pasado (tiempo de } \\
\text { entrega, calidad en el trabajo) }\end{array}$ \\
\hline & $\begin{array}{l}\text { Habilidad para terminar proyectos a } \\
\text { tiempo }\end{array}$ \\
\hline & Proyectos similares \\
\hline \multirow{4}{*}{ Técnicos } & $\begin{array}{l}\text { Disponibilidad de personal experto y } \\
\text { técnico }\end{array}$ \\
\hline & $\begin{array}{l}\text { Disponibilidad de recursos (capacidad } \\
\text { técnica) }\end{array}$ \\
\hline & Capacidad-carga de trabajo \\
\hline & $\begin{array}{l}\text { Conocimiento especializado en el método } \\
\text { de construcción }\end{array}$ \\
\hline \multirow{5}{*}{ Organizacionales } & Capacidad administrativa \\
\hline & Reputación \\
\hline & Salud y seguridad ocupacional \\
\hline & Calidad \\
\hline & Relación con los clientes \\
\hline \multirow{3}{*}{$\begin{array}{l}\text { Financieros y } \\
\text { económicos }\end{array}$} & Estabilidad financiera \\
\hline & Flujo de caja libre \\
\hline & Costo (precio de subasta) \\
\hline
\end{tabular}

\section{Métodos multicriterio de selección de contratistas}

La precalificación y la calificación de contratistas es un problema de decisión multicriterio que debe involucrar variables cualitativas y cuantitativas, en el cual son numerosas las partes interesadas y pueden tener impactos desde diferentes ópticas: económica, monetaria o social. Por lo anterior, se han desarrollado métodos que buscan realizar una efectiva selección de contratistas y que funcionan como marcos de referencia para llevar a cabo procesos de selección en múltiples instancias. Dichos métodos se basan en la utilización de información cuantitativa y/o cualitativa relevante asociada a los contratistas y al proyecto en cuestión, la cual es procesada y analizada con el fin de entregar resultados que sugieren cuál o cuáles son los contratistas mejor calificados y por lo 
tanto más adecuados para ejecutar el proyecto. En la Tabla 5 se observan los modelos más utilizados para el problema de selección de contratistas de proyectos de construcción e infraestructura en países Europeos, EEUU, China, India, Sudáfrica, Chile y Perú en los últimos 5 años.

Tabla 5: Modelos de selección de contratistas de la construcción (Jato-Espino et al., 2014)

\begin{tabular}{|c|c|c|c|}
\hline Enfoque & Método & $\begin{array}{c}\text { N. de } \\
\text { ocurrencias }\end{array}$ & $\begin{array}{l}\% \text { del } \\
\text { total }\end{array}$ \\
\hline \multirow{5}{*}{ Individual } & AHP & 20 & 22.73 \\
\hline & ELECTRE & 6 & 6.82 \\
\hline & TOPSIS & 3 & 3.41 \\
\hline & ANP/Delphi/GST & 2 & 2.27 \\
\hline & Otros & 1 & 1.14 \\
\hline \multirow{6}{*}{ Combinado } & AHP & 26 & 29.54 \\
\hline & FSS & 24 & 27.27 \\
\hline & TOPSIS & 11 & 12.50 \\
\hline & $\begin{array}{c}\text { ANP/MCS/MIVES/ } \\
\text { VIKOR }\end{array}$ & 4 & 4.54 \\
\hline & $\begin{array}{c}\text { COPRAS/GST/ } \\
\text { PROMETHEE/SAW }\end{array}$ & 2 & 2.27 \\
\hline & Otros & 1 & 1.14 \\
\hline
\end{tabular}

En términos de métodos, el más utilizado es el AHP, debido a su sencillez de aplicación y flexibilidad. Además, el AHP es una técnica fácilmente combinable con otros métodos, con frecuencia como un mecanismo para sopesar la importancia de los criterios que definen el problema de toma de decisiones. Mientras tanto, la razón por la cual los conjuntos borrosos SFS son utilizados como enfoque híbrido por su capacidad de tener en cuenta la incertidumbre y la imprecisión en los datos. Por otro lado, teniendo en cuenta los FSS más como un complemento de un método multicriterio por sí mismo. La técnica TOPSIS basado en el concepto de cercanía a las soluciones ideales $\mathrm{y}$ anti-ideales a un problema de toma de decisiones, es un método fácil de calcular y algorítmicamente estructurado, que automatiza considerablemente sus condiciones de ejecución. Del mismo modo, las búsquedas del método VIKOR para la solución más cercana al ideal general, pero a diferencia de TOPSIS, su proceso de normalización es hecho linealmente, en lugar de vectorialmente. En cualquier caso, la mayor diferencia reside en su grado de difusión; la propagación de VIKOR está lejos de la de TOPSIS, presumiblemente porque el primero se hizo conocido al público varios años después de la segunda (Jato-Espino et al., 2014).

\section{Contratación pública en obras de infraestructura en Colombia}

En Colombia, los procesos de contratación pública para proyectos de infraestructura están a cargo de la Agencia Nacional de Infraestructura ANI. Estos procesos se encuentran regulados por las leyes 80 de 1993, 1150 de 2007 y 1474 de 2011. En materia de infraestructura de transporte y en los demás aspectos se rigen por la ley 1682 de 2013 y por el derecho privado. Además, el decreto 1510 de 2013 por el cual se reglamenta la contratación pública. Para el caso específico de selección de contratistas para proyectos importantes de infraestructura la modalidad más usada es la Asociación Público Privada APP. El procedimiento de contratación se encuentra expuesto en la ley 1508 del 2012. Actualmente, Colombia viene desarrollando las denominadas carreteras de la prosperidad como eje fundamental para el desarrollo de la infraestructura del país. Para la adjudicación de dichos contratos, ANI está utilizando dentro de la licitación pública la modalidad de APP. Existen dos tipos de APP, una de iniciativa pública que es el Estado Colombiano quien convoca y otra es la de iniciativa privada, quien es una persona natural o jurídica de derecho privado quien estructura el proyecto por su propia cuenta y riesgo, asumiendo la totalidad de los costos de la estructuración. A su vez, esta última no podrá requerir más del $20 \%$ del presupuesto estimado de inversión del proyecto. La Figura 2 describe el proceso de adjudicación del contrato.

\section{Metodología del modelo propuesto}

Después de un análisis detallado del estado del arte con respecto a los métodos de análisis existentes para la contratación pública de proyectos de infraestructura, se identifica que los MCDM son un enfoque de apoyo en la evaluación y decisión operativa adecuado para hacer frente a problemas complejos que ofrecen una alta incertidumbre, diferentes formas de datos e información, intereses múltiples, sistemas complejos, como es el caso del problema de selección de contratistas para proyectos de infraestructura. En general, el problema MCDM para TDACI implica $m$ alternativas evaluadas en $n$ criterios. Así, la matriz de decisión agrupada se representa en (1).

Donde $x_{\mathrm{ij}}$ es el desempeño del criterio $j$-ésimo de la $i$-ésima alternativa, $w_{\mathrm{j}}$ es el peso del criterio $j, n$ es el número de 
Etapa 1: Precalificación

Invitación a precalificar
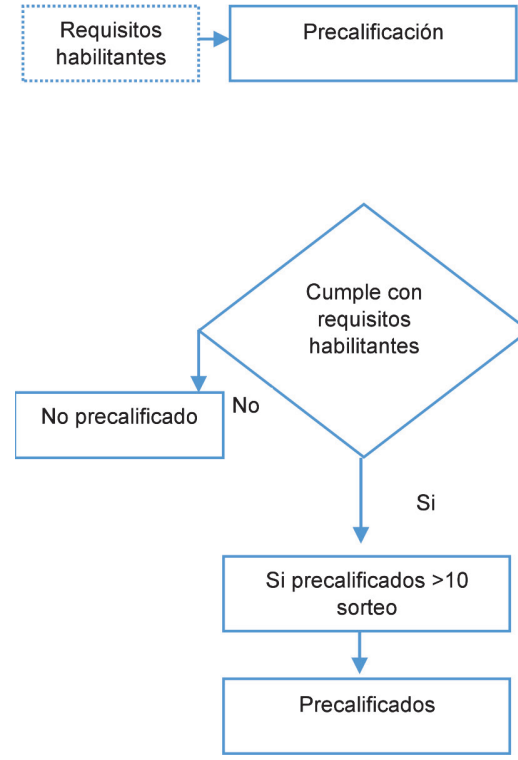

Etapa 2: Selección

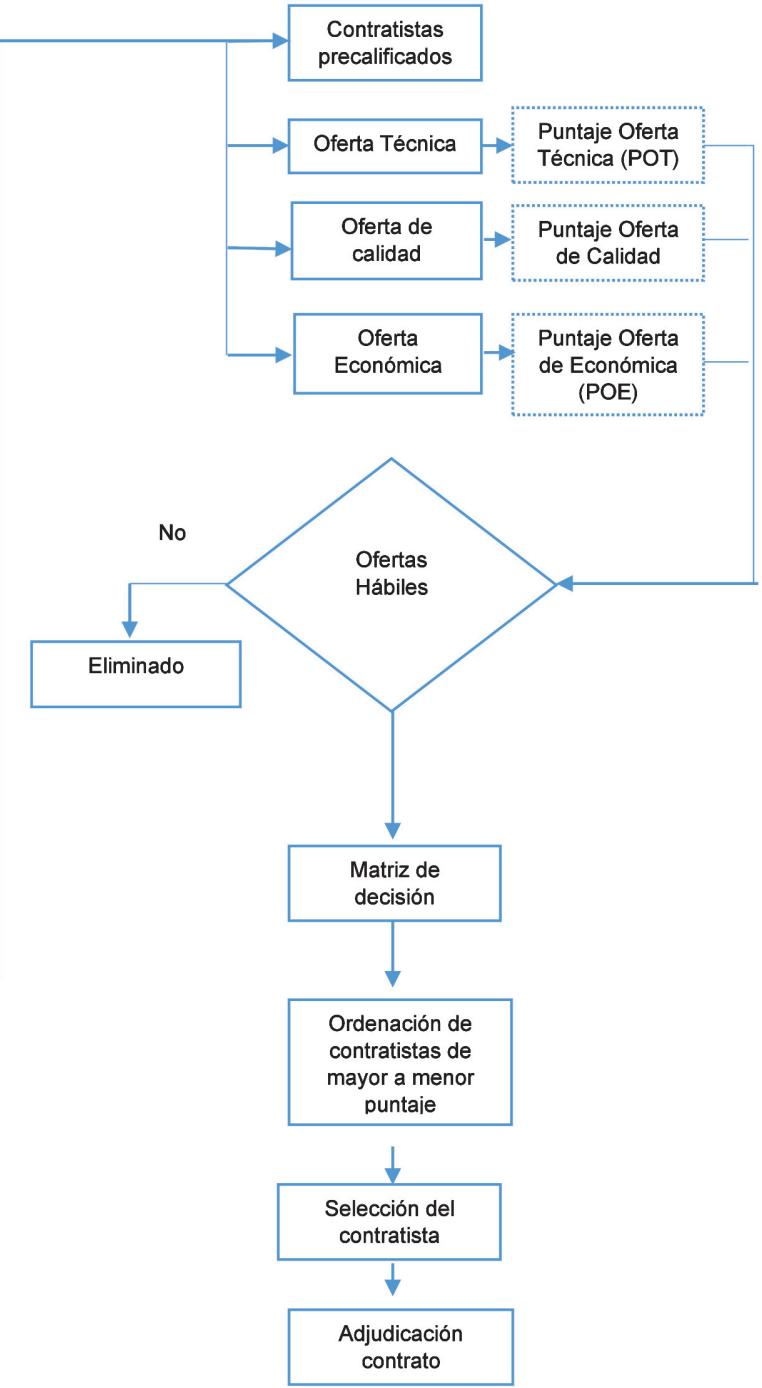

Figura 2: Proceso de la adjudicación de una APP de iniciativa pública en Colombia

$$
\begin{array}{ccccc}
\operatorname{criterios} & C_{1} & C_{2} & \cdots & C_{n} \\
(\operatorname{pesos} & w_{1} & w_{2} & \cdots & \left.w_{n}\right)
\end{array}
$$

alternativas - - - - - - - - - - -

$$
X=\begin{gathered}
A_{1} \\
A_{2} \\
\vdots \\
A_{m}
\end{gathered} \quad\left(\begin{array}{cccc}
x_{11} & x_{12} & \cdots & x_{1 n} \\
x_{21} & x_{22} & \cdots & x_{2 n} \\
\vdots & \vdots & \ddots & \vdots \\
x_{m 1} & x_{m 2} & \cdots & x_{m n}
\end{array}\right)_{m \times n}
$$

criterios y $m$ es el número de alternativas. El modelo propuesto tiene 3 etapas principales que contienen varios (1) pasos: i) etapa de determinación de criterios y asignación de pesos, ii) precalificación y iii) selección del contratista. Las tres etapas se relacionan entre sí, porque en su orden son una variable de entrada para la siguiente etapa; dicho de otra manera, una vez determinados los criterios y sus pesos, estos servirán de insumo para las etapas de precalificación y selección. A su vez la precalificación, además de determinar los contratistas que pasarán a la siguiente etapa de selección, generará una puntuación que es insumo en la etapa de selección. Un enfoque de selección efectiva debe integrar la precalificación como parte de cualquier ejercicio de selección, combinar este con el costo total de la oferta para generar una puntuación 
May, J., Botero, S. y Gonzalez-Ruiz, J. (2016). Obras y Proyectos $20,56-74$

Corregir
nsistencias en
matriz de

combinada que permita recomendar el contratista más competente (Topcu, 2004). En cada etapa (preselección y selección) se evaluarán criterios diferentes.

\section{Etapa I. Determinación de criterios y asignación de pesos}

Un conjunto de criterios se establece para la evaluación de selección de contratistas de acuerdo a la naturaleza del proyecto de infraestructura. El establecimiento de un adecuado sistema de criterios que reflejen los objetivos del proyecto, las necesidades de los clientes y todos los factores que influyen en el éxito de un proyecto es fundamental para la clasificación de los contratistas. De acuerdo al modelo se proponen criterios y subcriterios para la precalificación y criterios para la selección, siendo la precalificación un criterio en la selección. En muchos problemas de decisión, los criterios de decisión no tienen la misma importancia por lo que un peso que representa su importancia se asigna a cada criterio. Con una estructura jerárquica de criterios, cada criterio está asociado con un peso local y un peso global. El peso local de un criterio se refiere al peso en relación con otros criterios en el mismo grupo y nivel, lo que se evaluará mediante el proceso de comparación por pares. El peso global de un criterio se refirió a la importancia relativa de todos los demás criterios para el objetivo global del problema de decisión (NietoMorote y Ruz-Vila, 2012).

Para calcular el peso local de cada criterio, se requiere que los tomadores de decisiones proporcionen su juicio comparativo sobre la importancia relativa de un criterio sobre otro, pertenecientes ambos al mismo nivel y el grupo en la estructura jerárquica. Para determinar los pesos se utilizará la metodología de Proceso Analítico Jerárquico AHP que de acuerdo al estado del arte es la metodología más usada para la determinación de pesos a los criterios por su sencillez en su aplicación y porque es fácilmente combinable con otros métodos (Jato-Espino et al., 2014). Para la obtención de la información por parte de los expertos normalmente se utilizan técnicas tales como entrevistas. Los criterios utilizados pueden ser cualitativos o cuantitativos y la jerarquía de las alternativas se calcula teniendo en cuenta la contribución que cada criterio y subcriterio tiene en el resultado final. El modelo propuesto para la selección de contratistas para proyectos de infraestructura de iniciativa pública se describe en la Figura 3.

El AHP está basado en una estructura jerárquica de los elementos envueltos en el problema de decisión. El establecimiento de la jerarquía requiere conocimiento y experticia por parte del tomador de decisiones para cada problema específico. La jerarquía más simple se compone de 3 niveles (Anagnostopoulos y Vavatsikos, 2006). En el primer nivel se encuentra la meta, respecto a la cual se toma la decisión final; en el segundo nivel los criterios de selección, y en el tercer nivel los subcriterios o alternativas a evaluar. La flexibilidad del modelo permite hacerlo más complejo si así se requiere para determinado problema, añadiendo niveles adicionales con sub-metas, criterios y sub-criterios. Se deben realizar los siguientes pasos:

Paso 1. El proceso de jerarquización se basa en comparaciones por pares: El tomador de decisiones compara dos alternativas $A_{\mathrm{i}}$ y $A_{\mathrm{j}}$ respecto a un criterio y asigna un valor numérico para darles un peso relativo. La comparación se basa en una escala fundamental que puede variar, por lo cual se puede seleccionar entre alguna escala utilizada previamente o definir una nueva. La Tabla 6 muestra la escala de importancia relativa a 5 niveles.

Tabla 6: Escala para importancia relativa AHP (Saaty, 1995)

\begin{tabular}{|c|l|}
\hline $\begin{array}{c}\text { Valor } \\
\text { numérico }\end{array}$ & \multicolumn{1}{|c|}{ Descripción } \\
\hline 1 & Ambos criterios tienen igual importancia \\
\hline 3 & Débil o moderada importancia de uno sobre otro \\
\hline 5 & Importancia fuerte de un criterio sobre otro \\
\hline 7 & Importancia muy fuerte de un criterio sobre otro \\
\hline 9 & Importancia absoluta de un criterio sobre otro \\
\hline
\end{tabular}

Teniendo $n$ alternativas se debe obtener entonces una matriz de tamaño $n \times n$. Nótese que para cada nivel se construye una matriz cuadrada incluyendo todas las alternativas. Todos los elementos de la diagonal principal en una matriz de comparación deben tener valor 1 , es decir, $a_{\mathrm{ij}}=1$, lo anterior debido a que una alternativa tiene una importancia relativa respecto a sí misma de $100 \%$. Además, la matriz es simétrica y cada elemento es el inverso multiplicativo de su simétrico, es decir, $a_{\mathrm{ij}}$ x $a_{\mathrm{ji}}=1$, lo cual quiere decir que $a_{\mathrm{ij}}=1 / a_{\mathrm{ji}}$, de no ser así obviamente habría inconsistencia en la comparación ya que no se conservaría la importancia 


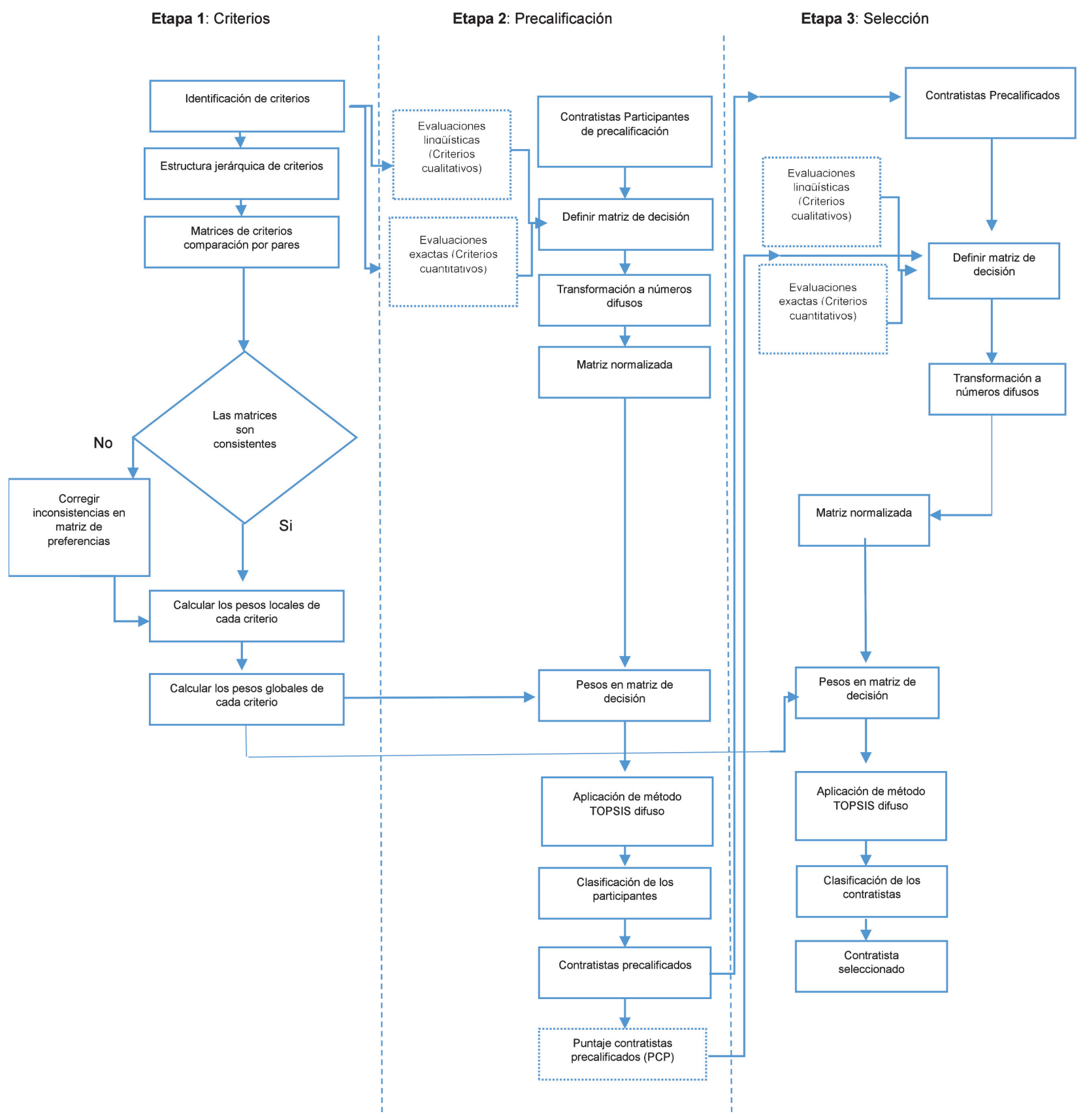

Figura 3: Modelo propuesto para selección de contratistas

relativa al realizar una comparación en dirección contraria.

La matriz de comparación por pares $A$, de las alternativas $P$, con respecto al criterio $K$, se expresa en (2).

$\begin{array}{ccccc}K & P_{1} & P_{2} & \ldots & P_{2} \\ P_{1} & 1 & a_{12} & \ldots & a_{12} \\ P_{2} & 1_{/ a_{12}} & 1 & \ldots & 1 \\ \vdots & \vdots & \vdots & \vdots & \vdots \\ P_{n} & 1_{/ a_{1 n}} & 1_{/ a_{2 n}} & \ldots & 1\end{array}$

Paso 2. Dada la matriz expresada en (2), se suman verticalmente los elementos de cada columna. Así se obtienen los valores de (3).

$v_{1}, v_{2}, \ldots, v_{n}=\sum_{1}^{n} a_{i}$

Una vez obtenida la suma de cada columna, se divide cada elemento de la matriz de comparaciones entre la suma obtenida, para conseguir la matriz de comparaciones normalizada (4) 


\section{Mayor, J, Boter, S. y Gonzalez-Ruiz, J. (2016). Obras y Proyectos $20,50-74$}

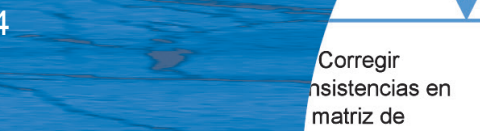

Paso 5. Posteriormente, el proceso se repite hasta terminar

$A_{\text {normalizada }}=\left(\begin{array}{cccc}1 / v_{1} & a_{12} / v_{2} & \ldots & a_{1 n} / v_{n} \\ a_{21} / v_{1} & 1 / v_{2} & \ldots & a_{2 n} / v_{n} \\ \cdots & \cdots & \cdots & \cdots \\ a_{n 1} / v_{1} & a_{n 2} / v_{2} & \cdots & 1 / v_{n}\end{array}\right)$

(4) Paso 6. A pesar de la experiencia y conocimiento que tenga el tomador de decisiones, puede haber comparaciones inconsistentes con otras. Una matriz de comparación es consistente si $a_{\mathrm{ij}} \mathrm{x} a_{\mathrm{jk}}=a_{\mathrm{ik}}$, para todo $i, j, k$. El AHP utiliza una medida de consistencia de los juicios realizados en las

Paso 3. Con la matriz de comparaciones normalizada, se calcula el vector columna que contiene los promedios de las filas como se expresa en (5).

$$
p=\left(\begin{array}{l}
\frac{1}{n} \sum_{1}^{n} a_{1 j} \\
\frac{1}{n} \sum_{1}^{n} a_{2 j} \\
\frac{1}{n} \sum_{1}^{n} a_{n j}
\end{array}\right)
$$

Se obtiene el vector de prioridades de los criterios, como se expresa en (6).

$$
p=\left(\begin{array}{c}
p_{c 11} \\
p_{c 12} \\
\cdots \\
p_{c 1 n}
\end{array}\right)
$$

Se puede comprobar que la suma de los elementos del vector prioridades sea igual a 1 .

Paso 4. Se multiplican la matriz de decisiones [A], con los vectores de prioridades de los subcriterios $[\mathrm{P}]$ respecto al criterio de jerarquía superior, obteniéndose [P'], como se muestra en (7).

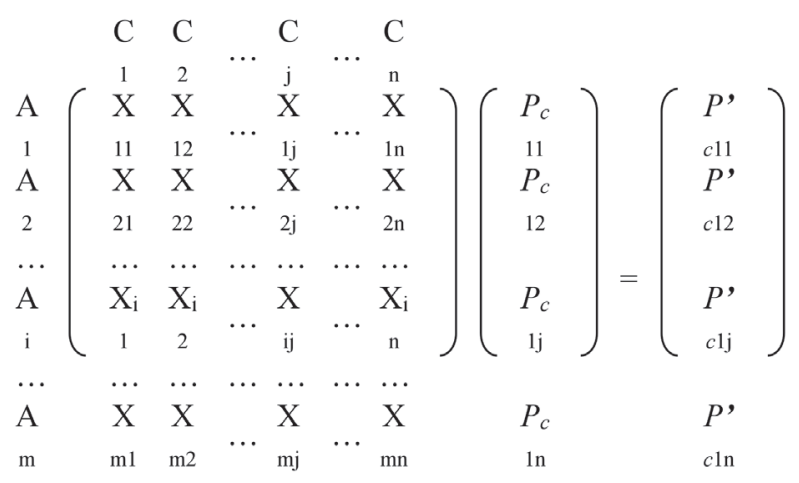
comparaciones, conocida como el Índice de consistencia o CI, y se calcula para cada matriz de comparación de acuerdo a los siguientes cálculos:

- Se divide el vector [P'] entre su correspondiente elemento en $[\mathrm{P}]$ obteniéndose $[\mathrm{D}]$.

- El promedio de los elementos de [D] (autovalor máximo) es $\lambda_{\text {max }}$. Luego, si $n$ es el tamaño de la matriz se aplica (8).

$$
C I=\frac{\lambda_{\max }-n}{n-1}
$$

- El índice de consistencia se divide entre el promedio de índice de consistencia aleatorio, para obtener la relación de consistencia o $\mathrm{CR}$, de acuerdo a (9).

$$
C R=\frac{C I}{I A}
$$

- El $I A$ en (9) es un valor constante para una matriz de $n \times n$, el cual se obtiene por medio de simulación de matrices de igual tamaño, teniendo en cuenta la misma escala de comparación, asegurando que $a_{\mathrm{ij}}=1 / a_{\mathrm{ji}}$

- Para el cálculo de $I A$, Saaty (1995) ha aproximado índices aleatorios IA para diversos tamaños de muestra $\mathrm{N}$ con base en diferentes ensayos, concluyendo que si el $C R$ es inferior a 0.1 , la matriz es consistente. La Tabla 7 muestra tamaños de la muestra.

Tabla 7: Índices aleatorios para diversos tamaños de muestra (Saaty, 1995)

\begin{tabular}{|c|c|c|c|c|c|c|c|c|c|c|c|c|}
\hline $\mathrm{N}$ & 1 & 2 & 3 & 4 & 5 & 6 & 7 & 8 & 9 & 10 & 11 & $\ldots$ \\
\hline IA & 0.00 & 0.00 & 0.58 & 0.90 & 1.12 & 1.24 & 1.32 & 1.41 & 1.45 & 1.49 & 1.51 & $\ldots$ \\
\hline
\end{tabular}


Paso 7. Se toman los pesos obtenidos de la evaluación de cada experto y se promedian, tanto para los criterios como para los subcriterios, estos serían los pesos locales. Para obtener los pesos globales de cada subcriterio en todo el sistema se multiplican los pesos globales por el peso del criterio.

\section{Etapa II. Precalificación}

Una tarea fundamental es evaluar la competencia del contratista con respecto a los criterios de precalificación, es decir, determinar quienes participarán para realizar la oferta técnica, de calidad y económica. Para ello es necesario definir la matriz de decisiones, especialmente cuando los criterios de decisiones pueden tener características cualitativas y cuantitativas. En el caso de criterios cualitativos es preferible que los tomadores de decisión para evaluar sus juicios utilicen términos lingüísticos en lugar de números reales. Por lo tanto, se proponen dos tipos de evaluaciones: 1) cuando el criterio de evaluación es cuantitativo, la evaluación son números reales, y 2) cuando el criterio de evaluación es cualitativo, la evaluación se realizará en términos lingüísticos. Las evaluaciones se describen subjetivamente en términos lingüísticos como muy bueno, bueno, aceptable, pobre, muy pobre. Estos términos se construyen para ayudar a quien toma las decisiones para evaluar la competencia de una alternativa en un criterio en particular. Para hacer frente al problema que estos términos no son matemáticamente operables, a cada término se le asocia un número difuso triangular.

En el caso de los criterios cuantitativos es necesario también convertir los números reales en números difusos. Para hacer frente a esta dificultad el número real que obtenga un contratista en un criterio determinado puede ser representado por un número difuso triangular. Por ejemplo (3.0, 3.0, 3.0) (Nieto-Morote y Ruz-Vila, 2012).

Para la clasificación de los contratistas se utilizará el método TOPSIS (Technique for Order Performance by Similarity to Ideal Solution), la cual fue desarrollado por Hwang y Yoon (1981), y se fundamenta en el concepto que la mejor elección entre diferentes alternativas es aquella que minimiza la distancia a la Solución Positiva Ideal PIS y a la vez maximiza la distancia a la Solución Negativa Ideal NIS, es decir, la mejor y peor soluciones teórica (Yalcin, et al., 2012). Se seleccionó este método porque de acuerdo a Roy y Słowiński (2013) para realizar la elección de un método de decisión multicriterio, es necesario analizar el tipo de resultados que espera que el método produzca, el tipo de resultados obtenidos es una característica que distingue a los distintos métodos. En el modelo propuesto, se espera que el resultado que se obtenga sea un puntaje de precalificación. Para este tipo de resultados Roy y Słowiński (2013) afirma que los métodos más pertinentes son los métodos basados en la teoría de valor del cual TOPSIS hace parte. De acuerdo al estado del arte, el método TOPSIS es uno de los más usados para resolver problemas de selección de contratistas de la construcción. Además, otra razón por la cual se seleccionó este método es debido que TOPSIS es flexible para combinarlo con lógica difusa, también útil para corregir problemas de arbitrariedad, incertidumbre o en algunos casos la indeterminación (Roy y Słowiński, 2013). En el método TOPSIS, los valores definidos como números triangulares borrosos, funciona como se muestra a continuación (Krohling y Campanharo, 2011).

Se construye la matriz de decisión $A^{\mathrm{k}}$ para el experto $k$, tal como lo muestra (10). Con $k=1,2, \ldots, l$; para un número de expertos $l$.

$$
A^{k}=\left(\begin{array}{ccc}
\tilde{x}_{11}^{k} & \cdots & \tilde{x}_{1 n}^{k} \\
\vdots & \ddots & \vdots \\
\tilde{x}_{m 1}^{k} & \cdots & \tilde{x}_{m n}^{k}
\end{array}\right)
$$

Donde $1,2, \ldots, m$ son las alternativas y $C_{1}, C_{2} \ldots C_{\mathrm{n}}$ son los criterios, $\tilde{x}_{i j}^{k}$ indica la puntuación de la alternativa $i$ para el criterio $C_{\mathrm{j}}$, de acuerdo al criterio de los expertos participantes y a la escala de valores definida para los números triangulares borrosos. Se debe encontrar una matriz promedio $A$, que no es más que el promedio de todas las calificaciones otorgadas por los expertos, tal como lo muestra la expresión (11).

$\tilde{x}_{i j}=\frac{\sum_{k=1}^{l} X_{i j}}{l}$

El vector de pesos $W=\left(\tilde{w}_{1}, \tilde{w}_{2}, \ldots, \tilde{w}_{n}\right)$ se compone de los pesos individuales $\tilde{w}_{j}(j=1,2, \ldots, n)$ para cada criterio $C_{\mathrm{j}}$. Después de la normalización, se procede a calcular la matriz normalizada ponderada $P=\left[\tilde{p}_{i j}\right]_{m \times n}$ con $i=1, \ldots$, 
$m$ y $j=1, \ldots, n$, multiplicando la matriz de decisión normalizada por el vector de pesos, como lo expresa la ecuación (12).

$$
\tilde{p}_{i j}=w_{i} \times \tilde{x}_{i j}
$$

Se identifican las soluciones positivas y negativas ideales, $A^{+}$y $A^{-}$(PIS y NIS), respectivamente, como lo expresa (13) y (14) respectivamente.

$$
\begin{aligned}
& A^{+}=\left(\max \tilde{p}_{i j} \mid j \in J, i=1, \ldots, m\right) \\
& A^{-}=\left(\min \tilde{p}_{i j} \mid j \in J, i=1, \ldots, m\right)
\end{aligned}
$$

Es decir, se construyen dos vectores con los valores máximos y mínimos de $p_{\mathrm{ij}}$ para cada alternativa, como lo expresan (15) y (16) respectivamente.

$$
\begin{aligned}
& A^{+}=\left(\tilde{p}_{1}^{+}, \tilde{p}_{2}^{+}, \ldots, \tilde{p}_{m}^{+}\right), \text {donde } p_{i j}=w_{i} \times \tilde{x}_{i j} \\
& A^{-}=\left(\tilde{p}_{1}^{-}, \tilde{p}_{2}^{-}, \ldots, \tilde{p}_{m}^{-}\right), \text {donde } p_{i j}=w_{i} \times \tilde{x}_{i j}
\end{aligned}
$$

Posteriormente, se calculan las distancias euclidianas a la solución positiva ideal $A^{+}$y a la solución negativa ideal $A^{-}$, respectivamente, como lo expresa (17) y (18).

$$
\begin{aligned}
& d_{i}^{+}=\sum_{j=1}^{n} d\left(\tilde{p}_{i j}, \tilde{p}_{j}^{+}\right), \text {para } i=1, \ldots, m . \\
& d_{i}=\sum_{j=1}^{n} d\left(\tilde{p}_{i j}, \tilde{p}_{j}\right), \text { para } i=1, \ldots, m .
\end{aligned}
$$

Donde $d$ corresponde a la distancia Euclidiana entre dos números triangulares. Se calcula el coeficiente de cercanía $C C_{\mathrm{i}}$ a la solución ideal, para cada alternativa $A_{\mathrm{i}}$. La expresión (19) corresponde a este coeficiente.

$$
C C_{i}=\frac{d_{i}}{d_{i}^{t}+d_{i}}
$$

Finalmente se ordenan las alternativas de acuerdo a su coeficiente de cercanía, teniendo en cuenta que las mejores alternativas son aquellas que presentan un mayor valor de dicho coeficiente y por lo tanto se encuentran más cerca de la solución ideal. Se puede determinar un umbral de puntaje para determinar los contratistas precalificados y no precalificados.

\section{Etapa III: Selección del contratista}

Una vez se tengan los contratistas precalificados y sus respectivos puntajes de precalificación, ellos realizarán la oferta de calidad, técnica y económica. Para la selección de contratistas se tomará el puntaje de cada uno obtenido en la precalificación y se ponderará con los puntajes que obtenga cada contratista para cada oferta. La clasificación de los contratistas se realizará por el método TOPSIS que seleccionará el contratista que más se acerque a la solución ideal detallado en la etapa II de precalificación. Para determinar si las ofertas económicas son hábiles o no, se usará la misma política gubernamental al respecto, es decir, si la oferta es mayor al $90 \%$ de la media de las ofertas económicas.

\section{Caso de estudio}

Al aplicar el modelo propuesto, el cual se muestra en la Figura 4, se utilizarán datos supuestos. El proyecto de infraestructura será una APP para el diseño, construcción, operación y financiación de una vía en Colombia. Por lo tanto, el problema se define en seleccionar el contratista más competente. Para lograr este objetivo, se realizarán tres etapas: 1 determinación de criterios y sus respectivos pesos, 2 precalificación y 3 selección.

\section{Etapa I - Determinación de criterios y pesos}

Para la asignación de los pesos para cada criterio y subcriterio se conformó un grupo de cuatro expertos de diferentes intereses, los cuales realizaron las comparaciones pareadas de todos los criterios de análisis. El grupo fue conformado así:

1. Un Ingeniero Civil, experto de una banca de inversión de estructuración de proyectos de infraestructura.

2. Un Ingeniero Administrador, docente investigador experto en temas relacionados a proyectos de infraestructura.

3. Un Abogado, miembro experto de la ANI.

4. Un Ingeniero Civil, miembro experto de una empresa contratista de proyectos de infraestructura

De acuerdo a la literatura analizada en el estado del arte y la anterior propuesta metodológica, se construyeron los criterios y sub-criterios. La Figura 5 muestra la jerarquía de los criterios y subcriterios. 


\section{Pesos de los criterios en la etapa de precalificación}

Para el caso de la precalificación se organizaron los subcriterios en criterios principales tales como experiencia, capacidad técnica, capacidad organizacional y la estabilidad financiera. La Tabla 8 muestra los criterios y sub-criterios de precalificación.

Para cada criterio establecido se determinaron su tipología de costo o beneficio y su dimensión; cuantitativo o cualitativo. Para el caso de este modelo se consideraron algunos subcriterios como cualitativos; sin embargo, podrían cambiarse a cuantitativos si se requirieran. Una vez identificados los criterios de decisión, se entrevistaron a 4 expertos, quienes realizaron comparaciones pareadas de criterios mediante la técnica AHP. Los pesos obtenidos para la precalificación después de aplicar los pasos 1-7 de la sección anterior, así como las ecuaciones (2) a (8), como se observa en la Tabla 9.

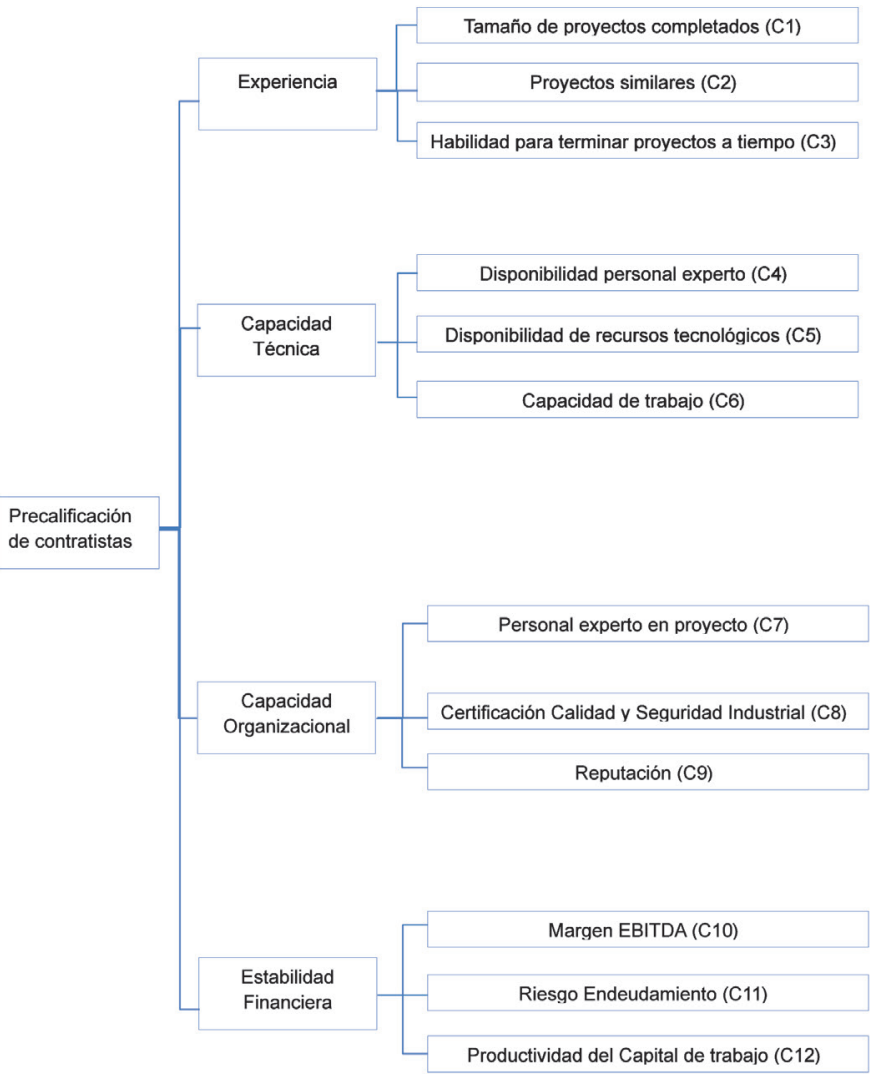

Figura 4: Estructura jerárquica de los criterios de precalificación

Tabla 8: Lista de criterios y sub-criterios en la precalificación

\begin{tabular}{|c|c|c|c|c|}
\hline Criterio & Sub-criterio & Descripción & Tipo & Dimensión \\
\hline \multirow{3}{*}{ Experiencia } & Tamaño de proyectos completados & $\begin{array}{l}\text { Tamaño de los proyectos ejecutados en los } \\
\text { últimos } 5 \text { años }\end{array}$ & Beneficio & Cualitativo \\
\hline & Proyectos similares & $\begin{array}{l}\text { Proyectos similares desarrollados en los últimos } \\
5 \text { años }\end{array}$ & Beneficio & Cualitativo \\
\hline & $\begin{array}{l}\text { Habilidad para terminar proyectos a } \\
\text { tiempo }\end{array}$ & $\begin{array}{l}\text { Proporción de proyectos ejecutados a tiempo en } \\
\text { los últimos } 5 \text { años }\end{array}$ & Beneficio & Cuantitativo \\
\hline \multirow{3}{*}{$\begin{array}{l}\text { Capacidad } \\
\text { Técnica }\end{array}$} & $\begin{array}{l}\text { Disponibilidad de personal experto y } \\
\text { técnico }\end{array}$ & Cantidad y grado de calificación del personal & Beneficio & Cualitativo \\
\hline & $\begin{array}{l}\text { Disponibilidad de recursos (Equipos, } \\
\text { tecnología) }\end{array}$ & $\begin{array}{l}\text { Evaluación del grado de suficiencia de los } \\
\text { equipos y tecnología }\end{array}$ & Beneficio & Cualitativo \\
\hline & Capacidad-Carga de trabajo & Capacidad de desarrollar nuevos proyectos & Beneficio & Cualitativo \\
\hline $\begin{array}{l}\text { Capacidad } \\
\text { Administrativa }\end{array}$ & $\begin{array}{l}\text { Personal experto en manejo de } \\
\text { Proyectos }\end{array}$ & $\begin{array}{l}\text { Personal que cuenta con certificación y } \\
\text { experiencia en administración de proyectos }\end{array}$ & Beneficio & Cualitativo \\
\hline \multirow{3}{*}{$\begin{array}{l}\text { Estabilidad } \\
\text { Financiera }\end{array}$} & Margen EBITDA & EBITDA/Ingresos & Beneficio & Cuantitativo \\
\hline & Riesgo de endeudamiento & Deuda Financiera/EBITDA & Costo & Cuantitativo \\
\hline & PKT & $\begin{array}{l}\text { Productividad del Capital de trabajo (KTNO/ } \\
\text { Ventas) }\end{array}$ & Costo & Cuantitativo \\
\hline
\end{tabular}


Tabla 9. Pesos de los criterios de precalificación

\begin{tabular}{|c|c|c|c|c|}
\hline Criterio & $\begin{array}{c}\text { Peso } \\
\text { criterio, } \\
\%\end{array}$ & Subcriterio & $\begin{array}{c}\text { Peso } \\
\text { local, } \\
\%\end{array}$ & $\begin{array}{c}\text { Peso } \\
\text { global, } \\
\%\end{array}$ \\
\hline \multirow{3}{*}{ Experiencia } & \multirow{3}{*}{25} & $\begin{array}{l}\text { Tamaño proyectos } \\
\text { completados }\end{array}$ & 42 & 10.43 \\
\hline & & Proyectos similares & 25 & 6.06 \\
\hline & & $\begin{array}{l}\text { Habilidad para terminar } \\
\text { proyectos a tiempo }\end{array}$ & 33 & 8.07 \\
\hline \multirow{3}{*}{$\begin{array}{l}\text { Capacidad } \\
\text { técnica }\end{array}$} & \multirow{3}{*}{18} & $\begin{array}{l}\text { Disponibilidad personal } \\
\text { experto }\end{array}$ & 45 & 8.14 \\
\hline & & Disponibilidad de recursos & 14 & 2.46 \\
\hline & & Capacidad de trabajo & 41 & 7.39 \\
\hline \multirow{3}{*}{$\begin{array}{l}\text { Capacidad } \\
\text { administra- } \\
\text { tiva }\end{array}$} & \multirow{3}{*}{11} & $\begin{array}{l}\text { Personal experto en } \\
\text { proyectos }\end{array}$ & 25 & 2.76 \\
\hline & & $\begin{array}{l}\text { Certificación calidad y } \\
\text { seguridad }\end{array}$ & 12 & 1.34 \\
\hline & & Reputación & 63 & 6.90 \\
\hline \multirow{3}{*}{$\begin{array}{l}\text { Capacidad } \\
\text { financiera }\end{array}$} & \multirow{3}{*}{46} & Margen EBITDA & 47 & 21.68 \\
\hline & & Endeudamiento & 27 & 12.39 \\
\hline & & Liquidez & 27 & 12.39 \\
\hline
\end{tabular}

\section{Pesos para los criterios de selección}

Para el caso de los criterios de selección se determinaron 4 criterios sin subcriterios: 1) puntaje precalificación; 2) oferta técnica; 3) oferta de calidad y 4) oferta financiera. Los criterios de selección se observan en la Tabla 10. Una vez identificados los criterios de decisión, se entrevistaron a 4 expertos, quienes realizaron comparaciones pareadas de criterios mediante la técnica AHP. Los pesos obtenidos para la selección, después de aplicar los pasos 1 a 7 de la metodología y las ecuaciones (2) a (8), se presentan en la Tabla 10.

Tabla 10. Criterios de selección

\begin{tabular}{|c|l|c|c|c|}
\hline Criterio & \multicolumn{1}{|c|}{ Descripción } & Tipo & Dimensión & $\begin{array}{c}\text { Peso, } \\
\%\end{array}$ \\
\hline $\begin{array}{c}\text { Precalificación } \\
\text { C1 }\end{array}$ & $\begin{array}{l}\text { Puntaje obtenido en la } \\
\text { precalificación }\end{array}$ & Beneficio & Cuantitativo & 38 \\
\hline $\begin{array}{c}\text { Oferta técnica } \\
\text { C2 }\end{array}$ & $\begin{array}{l}\text { El contratista realizará } \\
\text { una propuesta técnica } \\
\text { del proyecto (diseño } \\
\text { de trazado, puentes, } \\
\text { viaductos, túneles, } \\
\text { etc.) }\end{array}$ & Beneficio & Cualitativo & 14 \\
\hline $\begin{array}{c}\text { Oferta de } \\
\text { calidad C3 }\end{array}$ & $\begin{array}{l}\text { El contratista realizará } \\
\text { una propuesta de } \\
\text { mejoras adicionales }\end{array}$ & Beneficio & Cualitativo & 14 \\
\hline $\begin{array}{c}\text { Oferta } \\
\text { económica C4 }\end{array}$ & $\begin{array}{l}\text { Valor de la oferta } \\
\text { económica }\end{array}$ & Costo & Cualitativo & 34 \\
\hline
\end{tabular}

\section{Etapa II. Precalificación}

Para la precalificación se supondrá que existen 6 contratistas que están precalificando y a su vez, los criterios cualitativos están siendo evaluados por dos expertos. Para esta etapa se siguieron los pasos descritos en la metodología y las ecuaciones (9) a (19). Evaluar la calificación de las alternativas con respecto a cada criterio, dos sistemas de evaluación se definen: el uso de los números reales cuando el criterio tiene dimensión cuantitativa o usar términos lingüísticos cuando el criterio tiene dimensión cualitativa. En este último caso, cinco términos lingüísticos se utilizan para medir el nivel de rendimiento de cada contratista con respecto a cada criterio cualitativo. Estos términos lingüísticos con sus respectivos números difusos se definen en la Tabla 11.

Tabla 11. Variables lingüísticas para nivel de satisfacción de las alternativas (Wei et al., 2011)

\begin{tabular}{|c|c|}
\hline Variable lingüística & Número difuso \\
\hline Muy bueno & $(0.75,1.0,1.0)$ \\
\hline Bueno & $(0.50,0.75,1.0)$ \\
\hline Aceptable & $(0.25,0.5,0.75)$ \\
\hline Pobre & $(0.0,0.25,0.5)$ \\
\hline Muy pobre & $(0.0,0.0,0.25)$ \\
\hline
\end{tabular}

Las evaluaciones con términos lingüísticos de los contratistas con respecto a los criterios de evaluación cualitativa se transforman en los correspondientes números difusos. Para operar matemáticamente es necesario convertir los criterios cuantitativos que están en términos de números reales en números difusos también. Para hacer frente a esta dificultad el número real que obtenga un contratista en un criterio determinado puede ser representado por un número difuso triangular, por ejemplo (3.0, 3.0, 3.0) (Nieto-Morote y Ruz-Vila, 2012). Una vez aplicado todo el procedimiento, se obtuvieron los coeficientes de cercanía que se muestran en la Tabla 12.

Tabla 12. Clasificación contratistas en la precalificación

\begin{tabular}{|c|c|c|c|c|}
\hline Ranking & Alternativa & $d^{+}$ & $d^{-}$ & $C C_{i}$ \\
\hline 1 & 2 & 0.066 & 0.306 & 0.822 \\
\hline 2 & 3 & 0.108 & 0.264 & 0.709 \\
\hline 3 & 1 & 0.121 & 0.253 & 0.676 \\
\hline 4 & 4 & 0.141 & 0.231 & 0.622 \\
\hline 5 & 6 & 0.231 & 0.142 & 0.382 \\
\hline 6 & 5 & 0.307 & 0.065 & 0.175 \\
\hline
\end{tabular}


Para este caso aplicado sólo precalificarán los contratistas que obtengan un coeficiente de cercanía superior al $60 \%$. Para este caso se usará un umbral del $60 \%$, pero este podría ser superior. En este caso particular las alternativas 1, 2, 3 y 4 son los contratistas precalificados, es decir, que pasarán para la siguiente etapa de selección. A su vez el valor del coeficiente obtenido por cada uno, se convertirá en puntos para la etapa de selección, es decir que el contratista 1, por ejemplo, tendrá un puntaje de 68 puntos para la selección.

\section{Etapa III: Selección}

Para esta etapa ingresan como datos de entrada los pesos de los criterios de selección mostrados en la Tabla 10. Además, ingresan los contratistas precalificados, es decir, aquellos que obtuvieron un coeficiente de cercanía mayor al 60\%, mostrado en la Tabla 12. Se utilizarán las variables lingüísticas mostradas en la Tabla 11. La evaluación de los criterios cualitativos la realizaron dos expertos. Las calificaciones asignadas por los dos expertos para los criterios de dimensión cualitativa se muestran en la Tabla 13 y las puntuaciones de los criterios cuantitativos se muestran en la Tabla 14. En este caso todas las ofertas económicas se supondrán hábiles, de acuerdo a las disposiciones del gobierno, es decir, que superan el límite inferior que es el $90 \%$ a la media de las ofertas. Después de aplicar el mismo procedimiento de la precalificación, se obtiene la clasificación de las alternativas que se muestra en la Tabla 15.

Tabla 13. Calificaciones de criterios cualitativos de expertos en etapa de selección

\begin{tabular}{|c|c|c|c|c|}
\cline { 2 - 5 } \multicolumn{1}{c|}{} & \multicolumn{2}{c|}{ C2 } & \multicolumn{2}{c|}{ C3 } \\
\hline Alternativa & Experto 1 & Experto 2 & Experto 1 & Experto 2 \\
\hline 1 & Bueno & Bueno & Bueno & Muy bueno \\
\hline 2 & Muy bueno & Muy bueno & Muy bueno & Muy bueno \\
\hline 3 & Muy bueno & Muy bueno & Bueno & Bueno \\
\hline 4 & Muy bueno & Bueno & Aceptable & Bueno \\
\hline
\end{tabular}

Tabla 14. Calificaciones criterios cuantitativos

\begin{tabular}{|c|c|c|}
\cline { 2 - 3 } \multicolumn{1}{c|}{} & C1 & C4 \\
\hline Alternativa & Precalificación & Oferta económica \\
\hline $\mathbf{1}$ & 68 & 930 \\
\hline $\mathbf{2}$ & 82 & 917 \\
\hline $\mathbf{3}$ & 71 & 856 \\
\hline $\mathbf{4}$ & 62 & 884 \\
\hline
\end{tabular}

Tabla 15. Clasificación de contratistas en el proceso de selección

\begin{tabular}{|c|c|c|c|c|}
\hline Ranking & Alternativa & $d^{+}$ & $d^{-}$ & $C C_{i}$ \\
\hline 1 & 2 & 0.023 & 0.170 & 0.882 \\
\hline 2 & 3 & 0.080 & 0.115 & 0.591 \\
\hline 3 & 1 & 0.135 & 0.058 & 0.301 \\
\hline 4 & 4 & 0.162 & 0.031 & 0.159 \\
\hline
\end{tabular}

Por lo tanto, de acuerdo al caso supuesto el contratista que se debe seleccionar es la alternativa 2, quien es el que más se acerca al contratista ideal con un coeficiente de cercanía del $88 \%$.

\section{Análisis de sensibilidad}

Al sensibilizar los pesos de los criterios de selección de precalificación y oferta económica, en un $10 \%$, es decir, bajando el peso de precalificación de un 38 a un 34\% y la oferta económica subiendo en el mismo peso y manteniendo los otros dos criterios con los mismos pesos, se conserva la misma clasificación, aunque varía el $C C_{\mathrm{i}}$ (coeficiente de cercanía). La Tabla 16 muestra los resultados de la simulación.

Tabla16. Análisis de sensibilidad leve

\begin{tabular}{|c|c|c|c|c|}
\hline Ranking & Alternativa & $d^{+}$ & $d^{-}$ & $C C_{i}, \%$ \\
\hline 1 & 2 & 0.025 & 0.161 & 86.4 \\
\hline 2 & 3 & 0.074 & 0.114 & 60.5 \\
\hline 3 & 1 & 0.131 & 0.055 & 0.3 \\
\hline 4 & 4 & 0.153 & 0.032 & 17.5 \\
\hline
\end{tabular}

En este caso de estudio, al asignarle un peso a la precalificación de $10 \%$, la oferta económica del $65 \%$, la oferta técnica del $20 \%$ y la oferta de calidad del $5 \%$, el orden de clasificación de los contratistas cambiaría, colocando a la alternativa 3 como el número uno del ranking, esto es lógico dado que este contratista fue el que menor oferta económica realizó. La Tabla 17 muestra los resultados de la simulación.

Tabla 17. Análisis de sensibilidad fuerte

\begin{tabular}{|c|c|c|c|c|}
\hline Ranking & Alternativa & $d^{+}$ & $d^{-}$ & $C C_{i}, \%$ \\
\hline 1 & 3 & 0.024 & 0,110 & 82.3 \\
\hline 2 & 2 & 0.043 & 0.089 & 67.4 \\
\hline 3 & 4 & 0.081 & 0.052 & 0.39 \\
\hline 4 & 1 & 0.115 & 0.018 & 13.7 \\
\hline
\end{tabular}




\section{Conclusiones}

El nivel de éxito de cualquier proyecto de construcción depende en gran medida de la selección del contratista adecuado. Para evaluar la competencia de un contratista, el proceso de precalificación es ampliamente sugerido en la literatura revisada. En Colombia, este proceso sólo considera las variables de experiencia de inversión, su capacidad jurídica y la capacidad de endeudamiento y patrimonial, sin asignar una calificación al respecto y así como no considera criterios como desempeño pasado, personal experto, capacidad técnica y desempeño financiero; que de acuerdo al estado del arte son criterios fundamentales para el cumplimiento exitoso de un proyecto de infraestructura. Al no calificarse en la etapa de precalificación, se recurre a un sorteo para determinar los precalificados, cuando este procedimiento debería realizarse por méritos y no por suerte. A su vez, aunque el proceso aparentemente es muy objetivo, dado que no se consideran criterios cualitativos, se puede dejar de involucrar características cualitativas de los contratistas, que aportan para la determinación de la competencia del contratista. Otra debilidad del proceso es la asignación de los puntajes (pesos) para cada criterio, estos son asignados aleatoriamente y no se tienen en cuenta metodologías que involucren a expertos en determinado proyecto de infraestructura. Por último, aunque se corrige el problema de adjudicar el contrato al proponente con la oferta económica más baja por medio del límite inferior del $90 \%$ de la media, finalmente este último criterio es el que define el contratista ganador de la licitación, dado al gran peso asignado a este criterio, desconociendo los criterios de precalificación.

Para mitigar los problemas expuestos anteriormente, esta investigación propone un modelo de decisión multicriterio que se desarrolla en 3 etapas: 1) determinación de criterios y asignación de pesos; 2) precalificación y 3) selección del contratista. Para la asignación de los criterios y los pesos (ponderación) de los mismos se usó la metodología AHP y se contó con la participación de expertos en el área de infraestructura, los miembros fueron: un experto de la Agencia Nacional de Infraestructura ANI, un experto de una banca de inversión que estructura proyectos de Asociaciones Público Privadas APP, un experto de una empresa que participa en licitaciones de infraestructura por modalidad APP y un docente experto en proyectos de infraestructura.

Para las etapas de precalificación y selección se integró la teoría de conjuntos difusos con el método TOPSIS que combina un proceso de precalificación con selección, que de acuerdo al estado del arte, dicha combinación es más eficaz para seleccionar el contratista más competente. Esta competencia es medida a través de criterios tales como la experiencia, la capacidad técnica, la capacidad administrativa y la capacidad financiera. A su vez estos criterios tienen subcriterios de dimensión cualitativa y cuantitativa. El proceso de precalificación determina los contratistas que siguen el proceso de selección y a su vez asigna una calificación que se tiene en cuenta al momento de seleccionar el contratista. Dicha precalificación se basa en el concepto de que la mejor alternativa debe tener el mayor coeficiente de cercanía. En la etapa de selección se consideran los criterios de precalificación, oferta técnica, de calidad y económica. Finalmente se aplica la misma metodología de la precalificación y se selecciona el contratista que tenga el mayor coeficiente de cercanía con el contratista ideal. Al realizar un análisis de sensibilidad se observa que la metodología es sensible a un cambio fuerte en los pesos de los criterios de preselección y oferta económica, en el caso de estudio propuesto, el orden de la clasificación cambiaría cuando el peso de la precalificación es del $10 \%$, la oferta técnica es del $20 \%$, la de calidad el $5 \%$ y la oferta económica es del $65 \%$, en este caso particular la alternativa que ocupaba el segundo lugar pasaría al primero. Esto es lógico dado que dicha alternativa fue el que ofertó el precio más bajo.

El modelo propuesto es una herramienta de apoyo para los tomadores de decisiones, quienes se enfrentan a problemas de selección de contratistas en ambientes difusos y a su vez garantiza la selección por competencia y no por suerte. En esta misma línea, es una herramienta de doble vía, tanto para la selección como para el control del proyecto, dado el éxito en el cumplimiento de los proyectos se obtendrá un puntaje positivo en convocatorias futuras. Sin embargo, se aclara que utilizar este modelo, aunque mejora la eficacia de la contratación, este añade complejidad al procedimiento, haciendo que el proceso de selección probablemente se dilate en más tiempo por el cumplimiento del debido proceso, dado que podría presentarse mayores litigios por 
parte de los contratistas perdedores. Esta metodología sirve de referencia para aplicarlo en otros países y otros campos de selección. Debido a la gran cantidad de métodos de decisión multicriterio, sería útil aplicar este modelo con otros métodos para analizar los resultados obtenidos y validar e identificar las diferencias entre ellos. Además, se propone como trabajo futuro identificar metodologías que permitan a los estructuradores de proyectos determinar los requerimientos para las variables cuantitativas; tales como el valor de endeudamiento, valor de la carta de crédito y el valor patrimonial del oferente.

\section{Referencias}

Alhumaidi, H. (2015). Construction contractors ranking method using multiple decision-makers and multiattribute fuzzy weighted average. Journal of Construction Engineering and Management 141(4), 1-13

Anagnostopoulos, K.P. and Vavatsikos, A.P. (2006). An AHP model for construction contractor prequalification. Operational Research 6(3), 333-346

ANI (2013). Pliego de condiciones VJ-VE-IP-008-2013. Agencia Nacional de Infraestructura de Colombia

Arslan, G., Kivrak, S., Birgonul, M. T. and Dikmen, I. (2008). Improving sub-contractor selection process in construction projects: web-based sub-contractor evaluation system (WEBSES). Automation in Construction 17(4), 480-488

Awad, A. and Fayek, A.R. (2012). A decision support system for contractor prequalification for surety bonding. Automation in Construction 21, 89-98

Ballesteros-Perez, P., Carmen Gonzalez-Cruz, M. and CanavateGrimal, A. (2013). On competitive bidding: Scoring and position probability graphs. International Journal of Project Management 31(3), 434-448

Bastias, A. y Molenaar, K.R. (2010). Modelo de aprendizaje para la selección de un proyecto diseño-construcción (llave en mano) en el sector público. Revista Ingeniería de Construcción 25(1), 5-20

Bendana, R., del Cano, A. and de la Cruz, M.P. (2008). Contractor selection: fuzzy-control approach. Canadian Journal of Civil Engineering 35(5), 473-486

Bergman, M.A. and Lundberg, S. (2013). Tender evaluation and supplier selection methods in public procurement. Journal of Purchasing and Supply Management 19(2), 73-83
Bolton, P. (2009). The committee system for competitive BIDS in local government. PER: Potchefstroomse Elektroniese Regsblad 12(2), 57-96

Chou, J.S., Pham, A.D. and Wang, H. (2013). Bidding strategy to support decision-making by integrating fuzzy AHP and regressionbased simulation. Automation in Construction 35, 517-527

Darvish, M., Yasaei, M. and Saeedi, A. (2009). Application of the graph theory and matrix methods to contractor ranking. International Journal of Project Management 27(6), 610-619

DPN (2013). Decreto 1510 de 2013. Por la cual se reglamenta la contratación pública. Dirección de Planeación Nacional de Colombia. Diario oficial.

El-Abbasy, M.S., Zayed, T., Ahmed, M., Alzraiee, H. and Abouhamad, M. (2013). Contractor selection model for highway projects using integrated simulation and analytic network process. Journal of Construction Engineering and Management 139(7), 755-767

Estache, A. and Iimi, A. (2011). Bidders' entry and auctioneer's rejection: applying a double selection model to road procurement auctions. Journal of Applied Economics 14(2), 199-223

Hatush, Z. and Skitmore, M. (1998). Contractor selection using multicriteria utility theory: an additive model. Building and Environment 33(2), 105-115

Horta, I.M., Camanho, A.S. and Lima, A.F. (2013). Design of performance assessment system for selection of contractors in construction industry e-marketplaces. Journal of Construction Engineering and Management 139(8), 910-917

Hwang, C.L. and Yoon, K. (1981). Multiple attribute decision making. Springer Verlag, Berlin

Jaskowski, P., Biruk, S. and Bucon, R. (2010). Assessing contractor selection criteria weights with fuzzy AHP method application in group decision environment. Automation in Construction 19(2), 120-126

Jato-Espino, D., Castillo-Lopez, E., Rodriguez-Hernandez, J. and Canteras-Jordana, J. C. (2014). A review of application of multi-criteria decision making methods in construction. Automation in Construction 45, 151-162

Jato-Espino, D., Castillo-Lopez, E., Rodriguez-Hernandez, J. and Canteras-Jordana, J. C. (2014a). A review of application of multi-criteria decision making methods in construction. Automation in Construction 45, 151-162 
Krohling, R.A. and Campanharo, V.C. (2011). Fuzzy TOPSIS for group decision making: a case study for accidents with oil spill in the sea. Expert Systems with Applications 38, 4190-4197

Lambropoulos, S. (2007). The use of time and cost utility for construction contract award under European Union Legislation. Building and Environment 42(1), 452-463

Li, Y., Nie, X. and Chen, S. (2007). Fuzzy approach to prequalifying construction contractors. Journal of Construction Engineering and Management 133(1), 40-49.

Liu, H. and Yan, T. (2007). Bidding-evaluation of construction projects based on VIKOR method. IEEE International Conference on Automation and Logistics, Jinan, China, 17781782

MOP (2009). Decreto Supremo MOP No75 2009. Por la cual se establece el reglamento de contratos de obras públicas. Ministerio de Obras Públicas de Chile. Diario oficial.

Nassar, K. and Hosny, O. (2013). Fuzzy clustering validity for contractor performance evaluation: Application to UAE contractors. Automation in Construction 31, 158-168.

Nieto-Morote, A. and Ruz-Vila, F. (2012). A fuzzy multicriteria decision-making model for construction contractor prequalification. Automation in Construction 25, 8-19

Padhi, S.S. and Mohapatra, P.K.J. (2010). Centralized bid evaluation for awarding of construction projects - A case of India government. International Journal of Project Management 28(3), 275-284

Palaneeswaran, E. and Kumaraswamy, M. (2001). Recent advances and proposed improvements in contractor prequalification methodologies. Building and Environment 36(1), 73-87

Park, K. and Chul, L. (2012). Whole life performance bid evaluation in the Korean public sector. Journal of the Korea Institute of Building Construction 12(6), 682-700

Plebankiewicz, E. (2014). Modelling decision-making processes in bidding procedures with the use of the fuzzy sets theory. International Journal of Strategic Property Management 18(3), 307-316

Plebankiewicz, E. (2012). A fuzzy sets based contractor prequalification procedure. Automation in Construction 22, 433443
Plebankiewicz, E. (2009). Contractor prequalification model using fuzzy sets. Journal of Civil Engineering and Management 15(4), 377-385

Roy, B. and Słowiński, R. (2013). Questions guiding the choice of a multicriteria decision aiding method. EURO Journal on Decision Processes 1(1), 69-97

Saaty, T.L. (1995). Transport planning with multiple criteria: the analytic hierarchy process applications and progress review. Journal of Advanced Transportation 29(1), 81-126

San Cristóbal, J.R. (2012). Contractor selection using multicriteria decision-making methods. Journal of Construction Engineering and Management 138(6), 751-758

Singh, D. and Tiong, R.L.K. (2005). A fuzzy decision framework for contractor selection. Journal of Construction Engineering and Management 131(1), 62-70

Topcu, Y.K. (2004). A decision model proposal for construction contractor selection in Turkey. Building and Environment 39(4), 469-481

Trivedi, M.K., Pandey, M.K. and Bhadoria, S.S. (2011). Prequalification of construction contractor using a FAHP. International Journal of Computer Applications 28(10), 39-45

Turskis, Z. (2008). Multi-attribute contractors ranking method by applying ordering of feasible alternatives of solutions in terms of preferability technique. Technological and Economic Development of Economy 14(2), 224-239

Vahdani, B., Mousavi, S.M., Hashemi, H., Mousakhani, M. and Tavakkoli-Moghaddam, R. (2013). A new compromise solution method for fuzzy group decision-making problems with an application to the contractor selection. Engineering Applications of Artificial Intelligence 26(2), 779-788

Wang, D., Liu, X., and Liu, L. (2013). Bid evaluation behavior in online procurement auctions involving technical and business experts. Electronic Commerce Research and Applications 12(5), 328-336

Wang, W.C., Yu, W.D., Yang, I.T., Lin, C.C., Lee, M.T. and Cheng, Y.Y. (2013b). Applying the AHP to support the bestvalue contractor selection-lessons learned from two case studies in Taiwan. Journal of Civil Engineering and Management 19(1), 24-36 
Wang, J., Xu, Y. and Li, Z. (2009). Research on project selection system of pre-evaluation of engineering design project bidding. International Journal of Project Management 27(6), 584-599

Wang, J.J., Jing, Y.Y., Zhang, C.F. and Zhao, J.H. (2009b). Review on multi-criteria decision analysis aid in sustainable energy decision-making. Renewable and Sustainable Energy Reviews 13(9), 2263-2278

Watt, D., Kayis, B. and Willey, K. (2009). Identifying key factors in the evaluation of tenders for projects and services. International Journal of Project Management 27(3), 250-260

Wei,Z., Wang, X. and Guo, Q. (2011).Acontractor prequalification model based on triangular fuzzy number and TOPSIS. $18^{\text {th }}$ International Conference IEEE of Industrial Engineering and Engineering Management (IE\&EM), Changchun, 1-4
Yalcin, N., Bayrakdaroglu, A. and Kahraman, C. (2012). Application of fuzzy multi-criteria decision making methods for financial performance evaluation of Turkish manufacturing industries. Expert Systems with Applications 39, 350-364

Ye, K., Li, B. and Shen, L. (2013). Key factors considered in compiling tender prices for China's public works projects. Journal of Management in Engineering 29(3), 206-215 